\section{Revue critique de fixxion française \\ WX. contemporaine}

$23 \mid 2021$

Statut du personnage dans la fiction contemporaine

\title{
Parade et disparition du personnage postcolonial chez Kossi Efoui, Sandrine Bessora et Koffi Kwahulé
}

Marion Coste

\section{OpenEdition}

Édition électronique

URL : https://journals.openedition.org/fixxion/701

DOI : 10.4000/fixxion.701

ISSN : 2295-9106

Éditeur

Ghent University

\section{Référence électronique}

Marion Coste, « Parade et disparition du personnage postcolonial chez Kossi Efoui, Sandrine Bessora et Koffı Kwahulé ", Revue critique de fixxion française contemporaine [En ligne], 23 | 2021, mis en ligne le 15 décembre 2021, consulté le 17 février 2022. URL : http://journals.openedition.org/fixxion/701 ; DOl : https://doi.org/10.4000/fixxion.701

Ce document a été généré automatiquement le 17 février 2022.

\section{c) (†) $९$}

Les contenus de la Revue critique de fixxion française contemporaine sont mis à disposition selon les termes de la licence Creative Commons Attribution - Pas d'Utilisation Commerciale - Pas de Modification 4.0 International. 


\title{
Parade et disparition du personnage postcolonial chez Kossi Efoui, Sandrine Bessora et Koffi Kwahulé
}

\author{
Marion Coste
}

1 Il est devenu un lieu commun d'analyser dans les romans postcoloniaux les quêtes identitaires des personnages déchirés par leur appartenance à une africanité humiliée par la colonisation et à une occidentalité fantasmée. Lydie Moudileno ouvre d'ailleurs son ouvrage consacré aux romans congolais sur le constat d'une approche critique des littératures africaines comme témoignage d'une identité africaine authentique :

\begin{abstract}
En raison des circonstances particulières de son émergence sur la scène littéraire européenne, le texte africain a été soumis, dès les années 1920-1930, à un régime particulier dominé par l'attachement à une métaphysique de présence. [...] On le sait : il s'agissait, pour la première génération d'écrivains noirs des années 1930 à 1960, d'opposer aux constructions extérieures de l'Autre africain la manifestation d'une "âme noire" authentique dont les représentations négatives avaient jusque-là été prises en charge par l'imaginaire occidental ${ }^{1}$.
\end{abstract}

2 Cette lecture, motivée par un projet politique associé à la Négritude, octroie à la littérature un statut de témoignage (ce que Lydie Moudileno appelle "la théorie du reflet" ${ }^{\prime 2}$ et du même coup au personnage un statut de personne, capable de refléter les hantises identitaires des ex-colonisés et de leurs descendants.

Cette approche, que Lydie Moudileno reconnaît encore aujourd'hui, à la fois chez les écrivains et chez les commentateurs ${ }^{3}$, donne au personnage un rôle central, puisqu'il se charge d'incarner une façon d'être et de vivre (ou de subir) l'histoire, à la manière de Samba Diallo, le héros de L'aventure ambiguë de Cheikh Amidou Kane qui traduit le sentiment de déracinement et de déchirement de toute une génération d'intellectuels d'Afrique subsaharienne formés en Occident. Or, de nombreux critiques ont constaté que le roman contemporain français tend à l'effacement du personnage. Ainsi, Michel Biron, travaillant notamment sur Michel Houellebecq, souligne la tendance du personnage du roman moderne à la dépression, et le caractérise comme essentiellement "non conflictuel" ${ }^{4}$, l'opposant au héros du roman du XIX ${ }^{\mathrm{e}}$ siècle qui 
exerce sa volonté individuelle parce qu'il se heurte à une société injuste et rigide. Il déclare : “Mais la force de cet effacement n'en demeure pas moins une des

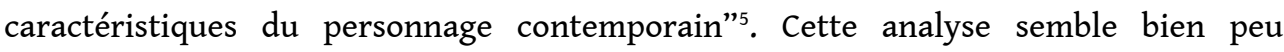
s'adapter à de nombreux romans postcoloniaux, chez qui le personnage est bien en conflit avec la société postcoloniale, et exerce une forme de puissance en tentant de s'y opposer : pensons par exemple à la révolte des nombreux personnages de La vie et demie de Sony Labou Tansi ou, plus récemment, aux personnages afropéens de Léonora Miano qui incarnent les enjeux identitaires qui se posent aux personnes d'ascendances subsahariennes vivant en Europe. Ces deux approches du personnage semblent donc contradictoires, et l'on pourrait imaginer que le personnage postcolonial échappe à cette tendance à la disparition. Pourtant, le personnage postcolonial est, dans certains cas, lui aussi affecté par une telle tendance. Ses contours s'estompent, jusqu'à interdire parfois l'illusion référentielle. Comment alors comprendre ce paradoxe? Le roman postcolonial s'éloigne-t-il de la problématique de l'identité par cette tendance à l'effacement?

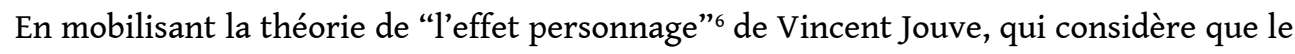
personnage est partiellement construit par les références culturelles du lecteur, et celle des parades postcoloniales de Lydie Moudileno, qui avance que les personnages postcoloniaux récents exhibent le caractère fabriqué et dynamique de l'identité, dans une volonté de refuser l'image fixe que l'occident peut se faire d'eux, nous présenterons les particularités du personnage postcolonial récent tel qu'il s'incarne dans cinq romans de Bessora, Kwahulé et Efoui.

5 Nous montrerons d'abord que ces personnages se caractérisent par une tendance à la parade, c'est-à-dire, selon la théorie de Moudileno, à la mise en scène de soi-même dans le but d'exhiber son pouvoir. En convoquant la théorie de "l'effet personnage" de Vincent Jouve, nous ferons l'hypothèse que cette hypermonstration de soi vient courtcircuiter une réception stéréotypée, pour ne pas dire raciste, des personnages racisés. On rejoint ainsi le deuxième sens de la parade, tel qu'il est utilisé par Moudileno: la parade est aussi une façon de parer les représentations fautives que le lecteur, habité par un imaginaire colonial, peut se faire des personnages racisés.

6 Nous analyserons ensuite la façon dont ces personnages implosent à la fin des romans : la superposition des masques de parade laisse in fine apercevoir la coquille vide que sont ces personnages. L'approche mimétique, qui voudrait que le personnage soit une personne non réelle à laquelle on peut s'identifier ou qui peut nous aider à comprendre les personnes qui nous entourent, semble alors impossible. En abordant la réception possible de ces romans, on peut cependant considérer, et ce sera notre deuxième hypothèse, qu'ils appellent à un dépouillement des identités narratives ${ }^{7}$ assignées aux personnes racisées pour leur permettre de s'inventer en s'identifiant aux personnages d'une littérature résolument postcoloniale ${ }^{8}$, c'est-à-dire qui met fin aux imageries et aux identités coloniales.

\section{Parade postcoloniale et "effet-personnage"}

7 Pour Vincent Jouve, le personnage se construit lors de la lecture, par la rencontre des indices textuels et des connaissances ou compétences du lecteur : "Il s'agira de montrer comment l'identité de l'être romanesque est le produit d'une coopération entre le texte et le lecteur. L'image mentale du personnage a en effet une spécificité qui la distingue 
aussi bien de la vision optique que de la représentation onirique"9. Vincent Jouve entre alors dans le détail du rôle du lecteur et de ses compétences, qu'il divise en "deux registres" ${ }^{10}$ : l'extratextuel, qui amène le lecteur à puiser dans son expérience de la vie pour compléter les vides du texte, et l'intratextuel, que le chercheur décrit ainsi :

Le second facteur qui influe sur l'image mentale est ce qu'on pourrait appeler l'“épaisseur intertextuelle". Du point de vue du lecteur, en effet, la figure romanesque est rarement perçue comme une créature originelle, mais rappelle souvent, de manière plus ou moins implicite, d'autres figures issues d'autres textes. Le personnage ne se réduit pas à ce que le roman nous dit de lui : c'est en interférant avec d'autres figures qu'il acquiert un contenu représentatif. S'il est donc exact que le lecteur visualise le personnage en s'appuyant sur les données de son expérience, cette matérialisation optique est corrigée par sa compétence intertextuelle. L'intertextualité du personnage est d'autant plus intéressante qu'elle a un champ d'action très large. Elle peut faire intervenir dans la représentation non seulement des personnages livresques (romanesques ou non), mais aussi des personnages fictifs non livresques (personnages de cinéma, par exemple), voire des personnages "réels", vivants ou non, appartenant au monde de référence du lecteur. ${ }^{11}$

8 Ces deux registres nous intéressent parce qu'il nous semble qu'ils expliquent une tendance des lecteurs, professionnels ou amateurs, à une conception réductrice et raciste des personnages postcoloniaux, ce que dénonce par exemple Léonora Miano :

Tout se passe, pour le lecteur occidental, dans la majorité des cas, y compris au sein de l'Académie, de manière à laisser penser que les personnes humaines représentées à travers les personnages romanesques ne sont que des objets d'étude distants, comme des animaux dans un zoo, qu'on peut trouver sympathiques, mais qui ne seront jamais des êtres dont l'expérience pourrait trouver un écho dans d'autres espaces que le leur. ${ }^{12}$

D'après l'autrice, si les personnages postcoloniaux sont perçus comme "des animaux dans un zoo", ce n'est pas à cause des éléments intratextuels, mais plutôt des images que peut convoquer le lecteur pour se représenter un personnage noir : ici, Léonora Miano dénonce un imaginaire colonial en évoquant les zoos humains des jardins d'acclimatation de la période coloniale. Cet imaginaire relève bien de ce que Vincent Jouve appelle "épaisseur intertextuelle", les personnes détenues dans des zoos étant bien "des personnages 'réels', vivants ou non, appartenant au monde de référence du lecteur". Les propos de Léonora Miano nous laissent entrevoir la dénonciation de la "coopération entre le texte et le lecteur", coopération qu'elle perçoit comme un jeu de dupe, dans lequel l'imaginaire colonial et raciste par lequel le lecteur occidental est fréquemment habité vient tordre les éléments textuels pour aboutir à une représentation conforme à des présupposés racistes.

La tendance à la parade que Lydie Moudileno observe chez les personnages postcoloniaux peut alors se comprendre comme une façon de saturer le texte d'indices sur le personnage, au point de paralyser toute compétence intertextuelle. En effet, pour l'autrice, les personnages paradent, c'est-à-dire qu'ils multiplient les détails visuels (habillement, démarche, expression), afin d'échapper à un regard qui projetterait sur eux un imaginaire colonial et raciste :

on pourrait proposer la définition suivante de la parade postcoloniale telle que nous l'aborderons : un acte de profération identitaire qui passe par une théâtralisation plus ou moins contrôlée - des corps et des images dans un espace particulier, et qui se pose en résistance à (ou en compétition avec) d'autres imaginaires et d'autres mises en scène auxquels le sujet substitue une auto-fiction dont il s'approprie le 
contrôle. Dans ce sens, la modalité de la parade traverse toute une production romanesque récente dans laquelle les identités ne sont plus données, mais défilent - et se défilent - dans une diversité étonnante de scénographies contemporaines. ${ }^{13}$

11 Étudions maintenant ces phénomènes d'hypermonstration dans Cyr@no de Bessora, Monsieur Ki et Babyface de Kwahulé et La Polka d'Efoui et voyons leurs répercussions sur l'effet-personnage produit.

12 L'hypermonstration dans Cyr@ano tient à ce que le personnage principal, Roxane, est habité d'un trop plein d'identités. La première phrase du roman est "Roxane? Vous vous appelez vraiment Roxane? Vous?"14 ${ }^{14}$ question posée par le jury d'un casting à Roxane, comédienne. Si son prénom arrête le jury, c'est parce que celui-ci renvoie à la Roxane de Cyrano de Bergerac : à son identité de personnage créée par Bessora, vient s'en ajouter une autre, créée par Rostand. Cette scène de casting est bien une parade, au sens dans lequel l'entend Lydie Moudileno, puisque le corps de Roxane, exposé par les projecteurs, est décrit avec minutie. Cette parade semble bien avoir pour objectif de déstabiliser un regard classifiant, qui tente de cerner en Roxane un genre et une race, sans succès.

C'est drôle, dit-il enfin, la couleur de vos yeux. Des lentilles?

Non, monsieur. Ces taches bleues éclaboussent mon œil droit depuis ma naissance.

De sorte qu'en effet il n'est pas tout à fait brun, cet œil-là.

L'autre, ajoute-t-il, n'est pas net non plus.

Mes yeux n'ont jamais voulu se déterminer.

Il faut avoir fait le tour d'un aquarium en quatre-vingts jours pour savoir que le principe d'indétermination est une anomalie dangereuse. Surtout dans les yeux. Les leurs donnent à lire des questions de poissons rouges, celles qu'on nie toujours s'être posées : d'où elle sort, celle-là ? quel âge elle peut avoir ? C'est une fille ou un transsexuel? C'est bizarre comme elle est foutue. Elle n'a pas de seins. Et ces cheveux qui vrillent sur sa tête, comment ils font pour tenir en l'air ? ( $C: 9)$

Ces "questions [...] qu'on nie toujours s'être posées" sont bien celles de la race, marquée par l'origine géographique supposée (d'où elle sort) et les cheveux crêpus (comment ils font pour tenir en l'air), de l'âge et du genre (fille ou transsexuel ? elle n'a pas de seins). On peut penser que l'indétermination dont jouit ici Roxane, si elle est due aux taches bleues de ses yeux et à un physique androgyne, est aussi la conséquence de la superposition des attributs de la Roxane de Rostand à celle de Bessora: le prénom ne correspond pas au personnage de Bessora, puisqu'il est associé à celui de Rostand.

À ce duo s'adjoint un troisième membre, lui-même dédoublé : Cyrano. En effet, Roxane entend en elle une voix répondant au nom de Cyrano. Ce Cyrano est bien un double du personnage d'Edmond Rostand, lui-même double d'une personne ayant réellement existé. Afin de séduire l'inconséquent Christian, dont Roxane (mais pas Cyrano) est tombée amoureuse, Roxane s'invente un double en ligne, qu'elle nomme Cyr@ano, et qui séduit Christian et se forge peu à peu une identité autonome, qui passe aussi par le registre visuel et la parade: armée d'une perruque et grâce à une mise en scène érotico-romantique dans un vieil hôtel parisien, Cyr@ano séduit Christian sans que celui-ci ne reconnaisse Roxane. On retrouve ici la situation du Cyrano d'Edmond Rostand, qui prête ses mots à Christian pour qu'il séduise Roxane. De même Cyr@ano séduit Christian pour le compte de Roxane. Ainsi, l'autrice amène le lecteur à penser une intertextualité qu'il n'aurait sans doute pas mobilisée spontanément pour lire Bessora, ou imaginer un personnage racisé. On peut faire l'hypothèse que l'autrice tend à prendre le contrôle de ce qui, dans l'analyse de Vincent Jouve, est à la discrétion du lecteur, c'est-à-dire "l'épaisseur intertextuelle". 
Babyface est aussi un personnage de parade, qui sature les perceptions des autres personnages et avec eux, on peut le supposer, celles des lecteurs et lectrices. Sa première apparition, lors d'une fête, est traduite par Mozati, une jeune femme qui s'éprendra de lui, et Jérôme, son vieil amant. La page est saturée de comparaisons : du côté de Mozati on trouve "comme un écolier" puis "comme un petit garçon"15. Jérôme déclare : "Il était là, devant moi, comme une apparition. Un jeune homme aux reflets timides et tristes. Presque un adolescent. Comme enfanté d'une pièce de théâtre russe du siècle dernier" (B 41). On apprend à la fin du roman que cette première apparition de Babyface a été préparée, et qu'ici le personnage parade afin de séduire Mozati. La parade lui permet en outre de se défaire de sa couleur de peau: la description de Jérôme, qui est blanc, fait imaginer un personnage de pièce russe ou un ange (apparition, reflets timides et tristes), soit des personnages blancs, alors que Babyface est noir. Ici encore, une intertextualité inattendue est suggérée, et permet peut-être de court-circuiter d'autres représentations, racistes, que le lecteur ou la lectrice occidental.e pourrait être tenté.e de mobiliser pour interpréter ce personnage.

Dans La Polka, c'est Nahéma, dite "la Polka", qui incarne le mieux cette logique de parade. D'abord, le personnage-narrateur la rencontre sous forme de photographie et de légende : elle est une jeune fille noire de la région du Pays du Lac qui danse la polka, sans qu'on sache exactement par quel moyen elle a pu apprendre cette danse exotique. Ensuite elle se matérialise mystérieusement lors d'une parade musicale du personnagenarrateur et de son ami Iléo, musiciens qui jouent de la rumba :

Iléo Para n'a pas compté ses deux temps deux mesures. Les doigts du bassiste sont restés collés au manche de sa contrebassine. On attend encore le fracas long fracas court des congas. Quelque chose d'autre est arrivé. Le ciel s'est soudain soulevé renversé, a jeté là cette jeune fille que nous avons tous reconnue : tête inclinée vers l'arrière, les cheveux tressés en fines antennes, le même sourire jeté vers le ciel d'où elle est tombée. Elle est là, déjà en piste, tournoyant avant même que la musique commence.

Dieu vous présent la Polka, hurle le maître de cérémonie ${ }^{16}$.

L'entrée en scène de Nahéma est une parade spectaculaire : elle devance son annonce par le maître de cérémonie, bouleverse jusqu'au ciel qui est "soulevé renversé", et dont elle semble tomber. La danse de Nahéma superpose sur le personnage toutes sortes de personnages ayant en commun d'être des femmes racisées fantasmées par un regard d'homme blanc :

Et sa danse est un déroulé de poses rapides et sans coupure : Balkis de Saba, reine d'Ethiopie dansant le Cantique des Cantiques, et chacun se sent fils aimé de David roi ; Kimpa Vita l'hérétique, la Dona Béatrice du Congo, celle qui s'est faite vierge noire et sainte qui a décidé de remettre au monde le Christ en terre Congo, qui a dérouté le roi Pedro IV du Portugal au point que sa langue a inventé le mot étrange de fausse saint Antoine pour la désigner, avant de prononcer contre elle et contre son ange gardien une sentence de mort par le feu. La voici ressuscitée dans le corps de la Polka. Et voici Bérénice l'amante, pieds menus dans ses sandales de reine d'Egypte, grands yeux ouverts sur Alexandrie au loin... ( $P: 35)$

Ces références à d'autres personnages de femmes racisées peuvent être comprises comme une façon d'expliciter la part d'intertextualité qui se met en place dans la construction de l'effet-personnage de Nahéma. Cette explicitation donne au lecteur la sensation que la spécificité de ce personnage lui échappe, d'autant plus que Nahéma, créant ces images par la danse, semble consciente des images qu'elle renvoie. Sa parade la protège ironiquement, en dénonçant la part de fantasme que le regard du lecteur ou 
de la lectrice risque de poser sur son corps dansant de femme noire. On assiste de nouveau à une surcharge de l'intertextualité par laquelle le personnage peut être appréhendé, ce qui paralyse la compétence intertextuelle à l'œuvre dans l'effetpersonnage de Vincent Jouve.

Les personnages de notre corpus sont donc bien des personnages de parade, à deux niveaux : d'abord, ils se mettent en scène, effectuant en cela une parade. Ensuite, cette mise en scène d'eux-mêmes est redoublée par une épaisseur intertextuelle très dense et très dirigée fournie par la voix narrative ou par un autre personnage. Ces deux modalités de parade permettent au personnage d'échapper à toute assignation, et surtout à une assignation raciale: si on essaie de les percevoir comme "effetpersonnage", on se rend compte qu'il est difficile de fixer une image, et tout particulièrement une couleur de peau. Cette indétermination forcée peut être comprise comme l'objectif de ces parades ambiguës.

\section{De la parade à la disparition}

Si ces personnages font preuve d'hypermonstration dans certains passages, ils semblent aussi affectés par une tendance à la disparition, que Michel Brion, et Dominique Rabaté $^{17}$ après lui, identifient comme caractéristique du personnage contemporain.

Le paradoxe n'est qu'apparent, puisque l'hypermonstration de la parade, nous l'avons $\mathrm{vu}$, tend à paralyser la réalisation entière d'un effet-personnage, privant le lecteur de l'exercice de sa compétence intertextuelle. Ces personnages, sans une participation pleine et entière du lecteur, sont alors fragiles. Ils sont même amenés à s'auto-annuler dans chacun des romans étudiés.

On assiste en effet dans nos cinq romans à de spectaculaires auto-anéantissements des personnages. Ainsi, Babyface, dont Mozati découvre qu'il lui a menti en prétendant être allé à Paris, explique :

Je ne m'appelle pas Babyface, ni Djê Koadjo. Je ne suis pas étudiant. Je n'ai jamais mis les pieds à Paris, je n'en ai même jamais éprouvé la démangeaison. Mon bizness ce sont les filles comme toi, Mozatimélédé, leur offrir l'homme dont elles n'osent même pas rêver. Avec toi je me suis montré le jeune homme timide et poli qui vit de profil. Avec une autre ç'aurait été un tout autre cinoche. Je ne suis ni timide ni poli. Je n'en suis pas le contraire non plus. Je ne suis rien. Rien d'autre que ce que vous, les femmes, créez. (B : 204)

Babyface n'est "rien", simple écran de projection des fantasmes, ici féminins. Babyface exemplifie peut-être dans ce passage le comportement du personnage postcolonial, tel que nous le découvrons dans notre corpus : assigné à porter les fantasmes avilissants ou au moins simplificateurs d'un regard extérieur, le personnage postcolonial se refuse à l'incarnation, et renvoie le lecteur à la fausseté de sa perception. Dans Désir de disparaître, Dominique Rabaté montre que le motif de la disparition est une forme de résistance, qui ressemble bien à celle de Babyface : "le roman constituerait [...] le lieu paradoxal de résistance face à la normalisation sociale, aux dispositifs toujours grandissants de contrôle et d'assignation, une façon de déserter qui puisse exprimer la force encore vitale d'une sécession individuelle" ${ }^{18}$. Babyface déserte bien le récit, et récuse ainsi le rôle qu'on voudrait lui faire jouer, ce "on" désignant bien sûr Mozati, mais aussi peut-être le lecteur. 

intégrité était vacillante puisqu'il oscillait entre le "je" et le "il" tout au long du roman. A la fin, ce personnage fait parler un pantin nommé ironiquement " $X$ " à sa place, et produit une description poétique de la ville ravagée par la guerre. Ce pantin est une première façon d'annuler le personnage, puisque sa voix est symboliquement associée à un être inanimé et innommé, répondant au non-nom d'X. De plus, ce pantin $X$ est une création de l'ami-jumeau, Iléo Para, qui a représenté le pirate noir de la BD Astérix : “C'est Iléo Para qui, un jour, a sorti des bandes dessinées ce petit bonhomme habillé d'une jupe de raphia qui trône tout en haut du grand mât des bateaux et crie Terre. Nous avons adopté le personnage sans nom qui parle ce petit nègre avaleur de $r$ comme transcrit dans les bulles" ( $P: 19)$. Ce pantin est présenté comme une fiction

Parce que s'il, monsieur Ki ou un autre, n'est pas venu pour Monsieur, alors il est venu pour vous, vu que vous êtes le seul Africain de l'immeuble, il est venu pour vous, j'ai fait le rapprochement vous comprenez, peut-être parce que vous ressemblez tellement à Monsieur, mais comme je dis, si ça se trouve, c'est que la solitude qui m'a raconté des histoires, enfin bon. Dites-moi, est-ce que je peux vous appeler Monsieur ? $^{19}$

confondu avec le narrateur de la bande magnétique, au point d'être nommé par un seul et même "Monsieur". La confusion repose sur un rapprochement raciste à peine voilé de la concierge, qui considère que l'oncle Koui, étant africain, ne peut venir voir qu'un Africain, et que le locataire actuel ressemble au locataire précédent, sans doute parce qu'il est Noir aussi. Si on ajoute que le premier locataire s'est suicidé, l'identification du second locataire au premier est de mauvais augure. La disparition du personnage est double : dans l'intrigue, tout semble annoncer son suicide à venir; dans l'écriture, le lecteur ou la lectrice ne sait plus dénombrer les différents personnages, et le titre peut se lire comme une interrogation (monsieur qui $?^{20}$ ).

Roxane, dans Cyr@no, est elle aussi anéantie : alors qu'elle tremble de se présenter à Christian, qu'elle a séduit sous l'identité de Cyr@no, cette dernière prend le contrôle de son être, et envoie l'intériorité de Roxane habiter une peinture réalisée par Christian. Roxane n'est plus qu'une image produite par Christian, qui fait figure de lecteur dans ce roman puisqu'il est régulièrement mis en scène en train de lire les messages de Cyr@no. Le personnage postcolonial Cyr@ano renonce à plaire à Christian, ce qui assure sa survie. Au contraire, Roxane, en continuant à désirer séduire Christian, se retrouve réduite à une projection de l'imaginaire de ce dernier : cette fin de roman, comme celle de Babyface, témoigne d'une révolte nécessaire et d'un refus catégorique d'être perçu à travers des stéréotypes réducteurs. 
coloniale, par sa tenue (la jupe de raphia), sa langue (le petit nègre) et son absence de nom. Le "je" s'effaçant derrière le pantin peut alors traduire l'effacement d'un personnage derrière les représentations racistes à travers lesquelles il risquerait d'être perçu. Les dernières lignes finissent d'effacer le "je" et avec lui le monde référentiel du roman : "Assis donc, avec ce rien de buste, de pas plus haut que le buste. Et je prends la pose - comme une seconde nature. Je n'ai plus envie ni d'aller ni de venir. Je n'aurai plus besoin d'arriver. Le monde se retire. Et je manque dorénavant" $(P: 156)$. Cette tendance du personnage à se dépouiller de lui-même est caractéristique de l'écriture de Kossi Efoui, et s'illustre à travers l'image du masque, très fréquemment convoquée, comme ici à la fin de La fabrique des cérémonies, pour décrire le corps d'un des personnages, qui s'est suicidé :

le masque du visage avec sa bouche attendant d'être arraché pareillement que le masque du tee-shirt, et alors on découvrirait non pas des muscles et des os mais encore le masque, avec la même expression de désintérêt fatal moulé dans une autre matière, gélatineuse ou friable, ainsi de suite, jusqu'à épuisement de toute matière capable de produire un quelconque avatar de ce mensonge que l'on nomme la face ou le visage, avec sa prétention à la vérité. Ce jeu drolatique de miroirs se réfléchissant l'un dans l'autre, démultipliant la même image qui bégaie à l'infini, jusqu'à son annulation, l'illusion de sa disparition ${ }^{21}$

Le phénomène de dépouillement qui aboutit à une "annulation" est ici traduit par la métaphore du masque: présenter le visage du personnage postcolonial comme un masque, comme une superposition de masques et l'impossibilité d'un visage véritable, c'est en révéler l'artificialité, dans une logique qui rejoint tout à fait la parade postcoloniale décrite par Lydie Moudileno.

Par ces scènes d'annulation, ces personnages interdisent ce que Jean-Marie Schaeffer appelle "activité projective", qu'il décrit ainsi : "Il existe une relation non contingente entre personnage fictif et personne [souligné dans le texte]: le personnage représente fictivement une personne, en sorte que l'activité projective qui nous fait traiter le premier comme une personne est essentielle à la création et à la réception des récits" ${ }^{22}$. Or, pour Jean-Marie Schaeffer, cette activité est essentielle dans l'intérêt que le lecteur prend à sa lecture : "Et dans la plupart des cas, une partie non négligeable du plaisir esthétique du lecteur réside justement dans cette activité projective" ${ }^{23}$. Il nous semble cependant que ces personnages, qui se vouent à l'auto-destruction, sont pourtant ceux qui concentrent l'intérêt du lecteur et retiennent l'attention de la critique ${ }^{24}$. Le fait que, dans notre corpus, chacun de ces personnages est éponyme confirme cette hypothèse. Ceci se comprend si l'on considère que ce que ces scènes d'annulation interdisent, c'est un rapprochement de ces personnages avec des personnes stables, dont le caractère serait appréhendé comme idem (identité-mêmeté, ce qui fait qu'on identifie une identité en ce que ses différentes apparitions se ressemblent ${ }^{25}$ ), pour le dire avec les concepts de Ricœur. Au contraire, ces scènes d'annulation peuvent être considérées comme des scènes de dépouillement, dans lesquelles l'idem s'efface, mais où l'ipse perdure : il ne s'agirait pas, pour le personnage, de signifier sa non-existence, mais de signifier que son existence transcende ce que le roman peut dire de lui, c'est-à-dire, dans nos exemples, les stéréotypes à travers lesquels le regard d'autrui est capable de l'appréhender et de lui donner une cohérence dans l'acte que Ricoeur nomme "refiguration" ${ }^{26}$. C'est justement lorsque cette cohérence implose, lorsqu'une action du personnage surprend au point de remettre en question son idem, que l'ipse se fait jour. Ces scènes de dépouillement, loin d'interdire l'activité projective, la renforceraient, puisque, d'après Jean-Marie Schaeffer, la différence entre un personnage et une 
personne tient au fait que cette dernière ne se réduit pas à ce "qu'on peut raconter à son sujet": "alors qu'une personne réelle est toujours ontologiquement irréductible aux récits (factuels) qu'on peut raconter à son sujet, un personnage fictif se réduit à ce que l'auteur en dit (ou à ce que l'acteur en présente)"27. Cette identité qui dépasse ce qu'on peut dire de la personne peut être rapprochée de l'ipse de Ricœur. Elle aurait pour intérêt, dans le cadre de nos romans, de laisser advenir l'impensable, ou du moins l'impensé : l'être hors de toute référence raciale.

31 L'effacement de nos personnages serait alors un dépouillement par lequel le soi ferait peau neuve, et s'ouvrirait à la possibilité d'une nouvelle identité narrative (c'est-à-dire une identité en tant qu'elle est racontée, qu'elle se construit dans et par un récit, autorécit ou récit par autrui), qui n'est pas assumée par ce récit. Voici comment Ricoeur présente les identités narratives :

Selon la compréhension intuitive que nous avons de cet état de choses, ne tenonsnous pas les vies humaines pour plus lisibles lorsqu'elles sont interprétées en fonction des histoires que les gens racontent à leur sujet? Et ces histoires de vie ne sont-elles pas rendues à leur tour plus intelligibles lorsque leur sont appliqués des modèles narratifs - des intrigues - empruntés à l'histoire proprement dite ou à la fiction (drame ou roman) ? Il semblait donc plausible de tenir pour valable la chaine suivante d'assertions: la compréhension de soi est une interprétation; l'interprétation de soi, à son tour, trouve dans le récit, parmi d'autres signes et symboles, une médiation privilégiée; cette dernière emprunte à l'histoire autant qu'à la fiction, faisant de l'histoire d'une vie une histoire fictive, ou, si l'on préfère, une fiction historique, entrecroisant le style historiographique des biographies au style romanesque des autobiographies imaginaires. ${ }^{28}$

Nous intéresse dans cette définition le fait que l'identité narrative comprend un double mouvement : d'une part, l'identité devient lisible lorsqu'elle se met en récit; d'autre part, pour atteindre à cette lisibilité, elle s'inspire d'autres récits, histoire ou fiction. Cela permet de comprendre le lien entre l'écriture des identités romanesques et les possibilités d'être offertes aux lecteurs et lectrices, particulièrement à celles et ceux qui sont racisé.e.s. Si les seuls récits disponibles, historiques ou fictionnels, présentent des personnages racisés humiliés et rabaissés, alors la construction de l'identité narrative des personnes racisées est compliquée.

On comprend donc le besoin de révéler le mensonge de ces identités narratives, afin d'inviter à de nouvelles "refigurations", la refiguration étant, d'après Ricoeur, la façon dont le lecteur construit, par sa réception, le récit, en fonction des stratégies mises en œuvre lors de la configuration, c'est-à-dire de la mise en intrigue. Éviter une refiguration simpliste, qui se contenterait de répéter des récits de personnages racisés rabaissants, serait alors la stratégie narrative élaborée par nos auteurs et autrice, grâce à une parade menant à la disparition. Cela rejoint la théorie des "dramaturgies noires" élaborée par Virginie Soubrier, qui repose sur l'identité d'un type de personnage qu'elle nomme "l'improviste", et qui est pour elle caractéristique du théâtre de Kwahulé :

Venu d'ailleurs, dépourvu de rôle prédéterminé, l'improviste est un personnage du seuil, à la fois dedans et dehors. Il est le tiers qui permet le récit, l'aveu, le témoignage et agit comme une force émancipatrice: en sa présence les autres personnages se dévoilent, déposent leur culpabilité et expriment leurs désirs. À cet égard, l'improviste est conçu comme un personnage heuristique, qui permet le dévoilement des psyché occidentale et africaine, masculine et féminine. Il témoigne ainsi du refus d'une certaine idée du drame, conçu comme une image totalisante du monde. Sa puissance de déclosion se manifeste à son apparition, à l'évitement du 
dialogisme traditionnel mais aussi à la catastrophe finale. Cette dernière est tout à

la fois la fin d'un monde et une genèse. ${ }^{29}$ contrairement aux autres personnages de notre corpus, ne prend jamais en charge la narration, sauf lorsqu'il s'adresse à Mozati dans l'extrait cité plus haut. Il est seulement perçu par autrui, qui reconnaît en lui ses fantasmes: il fonctionne bien comme "dévoilement des psyché". Il conduit à la "catastrophe finale" qui interdit toute clôture de l'intrigue et signe la fin du monde qu'avait jusque-là construit le roman. Comprendre cette catastrophe comme une genèse, c'est comprendre l'anéantissement du personnage de Babyface comme la possibilité qu'advienne une identité nouvelle, libérée du regard d'autrui.

\section{Conclusion}

Par l'usage de la parade, le personnage postcolonial exhibe la façon dont il est perçu à travers des stéréotypes racistes et réducteurs en saturant toute possibilité d'exercice de la "compétence intertextuelle" telle qu'elle est présentée par Vincent Jouve. Cette parade révèle l'artificialité du personnage, qui ne peut plus être appréhendé comme une personne, ce que mettent en exergue les scènes d'auto-destruction qui concluent nos cinq romans. Ce refus d'être du personnage postcolonial est pourtant à appréhender, à un autre niveau, à travers l'activité projective décrite par Jean-Marie Schaeffer : en refusant d'être ce qu'on voit en lui, le personnage postcolonial invite ses lecteurs et lectrices, et particulièrement celles et ceux qui sont racisé.e.s, à s'inventer hors des récits encore habités par des imaginaires coloniaux et racistes. La problématique de l'identité, d'après notre analyse, ne perd rien de son importance dans ces littératures, mais emprunte de nouvelles voies, prenant acte de l'influence de la fiction sur la réalité, cherchant à réinventer un imaginaire propice au déploiement de toutes les potentialités identitaires des lecteurs et lectrices.

\section{NOTES DE FIN}

1. Lydie Moudileno, Parades postcoloniales, la fabrication des identités dans le roman congolais, Paris, Karthala, 2006, p.10.

2. Ibid., p. 11.

3. "La responsabilité d'une critique mimétique de la littérature doit donc être renvoyée à la fois aux auteurs et à leurs critiques souvent avides de lire dans chaque texte le reflet fidèle de ce que le sujet (post)colonial trouvait sur ce chemin. Malgré une évolution remarquable du champ littéraire ces vingt dernières années, ce double jeu d'attentes continue à déterminer globalement la production, la réception et la diffusion du texte africain.", ibid., p. 12.

4. Michel Biron, "L'effacement du personnage contemporain”, Études françaises, vol. 41, no 1, p. 29.

5. Ibid., p. 40. 
6. Vincent Jouve, "Pour une analyse de l'effet-personnage", Littérature, $\mathrm{n}^{\circ} 85$, "Forme, difforme, informe", p. 103-111.

7. Paul Ricœur, Temps et récit, t.3, Paris, Seuil, 1983. L'hypothèse des identités narratives est ensuite reprise dans Soi-même comme un autre, Paris, Seuil, 1990.

8. On serait tenté de l'appeler "décoloniale", dans le sens où nous faisons l'hypothèse d'une littérature qui défait les traces de la colonialité dans le présent. Cependant, ce terme étant surtout rattaché à l'étude des cultures de l'Amérique latine, nous choisissons de conserver le terme "postcolonial", en lisant en lui une capacité au dépassement de la colonialité.

9. Vincent Jouve, op. cit., p. 109.

10. Ibid., p. 109-110.

11. Ibid., p. 110.

12. Léonora Miano, Habiter la frontière, Paris, l'Arche, 2012, p. 42-43.

13. Lydie Moudileno, op. cit., p. 23.

14. Bessora, Cyr@no, Paris, Belfond, 2011, p. 7 (dorénavant C).

15. Koffi Kwahulé, Babyface, Paris, Gallimard, 2006, p. 41 (dorénavant B).

16. Kossi Efoui, La Polka, Paris, Seuil, 1998, p. 34 (dorénavant $P$ ).

17. Dominique Rabaté, Désirs de disparaître. Une traversée du roman contemporain, Rimouski, Tangence, 2015.

18. Ibid., p. 20

19. Koffi Kwahulé, Monsieur Ki, Paris, Gallimard, 2010, p. 146 (dorénavant K).

20. Sur cette confusion des narrateurs, on renvoie à l'article de Romuald Fonkua, "Le corps de Monsieur Ki, une scénographie de la littérature", dans Sylvie Chalaye (dir.), Koffi Kwahulé, Paris, Garnier, 2019.

21. Kossi Efoui, La fabrique des cérémonies, Paris, Seuil, 2001, p. 250-251.

22. Jean-Marie Schaeffer, "Personnage", dans Oswald Ducrot et Jean-Marie Schaeffer (dirs), Nouveau dictionnaire encyclopédique des sciences du langage, Paris, Seuil,1995 [1972], p. 755.

23. Ibid., p. 755

24. Nous renvoyons par exemple aux travaux de Virginie Soubrier sur le personnage de "l'improviste", présenté plus loin.

25. "La mêmeté est un concept de relation et une relation de relations. En tête, vient l'identité numérique: ainsi, de deux occurrences d'une chose désignée par un nom invariable dans le langage ordinaire, disons-nous qu'elles ne forment pas deux choses différentes mais "une seule et même" chose. Identité, ici, signifie unicité : le contraire est pluralité (non pas une mais deux ou plusieurs); à cette première composante de la notion d'idenité correspond l'opération d'identification, entendue au sens de réidentification du même, qui fait que connaître c'est reconnaitre la même chose deux fois, n fois.", Paul Ricoeur, Soi-même comme un autre, op. cit., p. 140-141.

26. Paul Ricoeur, Temps et récit, Paris, Seuil, 1985, p. 87.

27. Jean-Marie Schaeffer, op. cit., p. 754.

28. Paul Ricoeur, Soi-même comme un autre, op. cit., p. 138.

29. Virginie Soubrier, “Une dramaturgie noire?”, dans Sylvie Chalaye (dir.), op. cit., p. 25.

\section{RÉSUMÉS}

Le personnage du roman postcolonial contemporain est affecté d'une tendance à la disparition qu'il partage avec l'ensemble des personnages de la littérature française contemporaine. Cependant, sa disparition est particulière parce qu'elle passe par une paradoxale hypermonstration, telle que la présente Lydie Moudileno sous le terme de "parade". Cette 
hypermonstration permet de court-circuiter une réception stéréotypée du personnage en saturant ses représentations. Je proposerai l'idée que cette parade constitue, dans cinq romans de Bessora, Kwahulé et Efoui, une première étape vers une disparition qui permettrait d'offrir au lecteur ou à la lectrice la possibilité d'inventer de nouvelles identités narratives, selon le concept de Ricoeur, débarrassées de tout stéréotype colonialiste.

INDEX

Mots-clés : personnage postcolonial, parade, disparition, identité narrative, réception

\section{AUTEURS}

MARION COSTE

CY Cergy Paris Université 